\title{
A new solar flare scenario: - High-beta plasma disruption -
}

\author{
Kiyoto Shibasaki \\ Nobeyama Solar Radio Observatory, Minamisaku, Nagano 384-1305, Japan \\ email: shibasaki@nro.nao.ac.jp
}

\begin{abstract}
The solar corona is believed to be generally low beta. In a low-beta plasma, energy is mainly stored as magnetic energy or current. To dissipate the stored magnetic energy in the highly conductive corona, anomalous resistivity is needed. Activities of outer layers of $\mathrm{X}$-ray loops in solar flares have been interpreted as the result of reconnection of magnetic field due to anomalous resistivity in a current sheet located above the flaring loop.

However, recent high-cadence EUV observations show many flares without opening up of magnetic field. This means that existence of current sheets above flaring loops is not a necessary element for flares. It is also found for many flares that low temperature prominence plasma are heated in the very early phase of lifting. These observational facts require a new solar flare scenario different from reconnection.

Recent multi-wavelength high-spatial and -temporal resolution observations show that flares originate in the lower atmosphere. We can expect high beta plasma and small curvature magnetic loops in the lower atmosphere. It is also observed high-velocity flows along magnetic loops associated with flares. Bounded motions of charged particles (both thermal motion and flow) along the magnetic lines of force create the centrifugal force. They exceed gravity force under certain conditions (small curvature and high velocity). They can even exceed magnetic tension force depending on beta values of the plasma in the loop.

In the case of the upward acceleration (centrifugal - gravity $>0$ ), the plasma is balanced by the magnetic tension force if the beta value is not large enough. This is a favorable condition for the interchange instability. Finite beta (beta $\sim 0.1$ ) plasma in a loop, whose ends are anchored at the photosphere, is unstable against the localized interchange (BALLOONING) mode. It can develop into non-linear phase and explosive phenomena are expected. This is called HIGH-BETA DISRUPTION. The high-beta disruption has many common characteristics to solar flares. Various phenomena related to solar flares such as, high-energy particle acceleration, plasma turbulence, plasma ejections, plasma heating, are interpred as the result of high-beta disruption. High spatial and temporal resolution observations of flares at various wavelengths are used to see the actual instability process. Shibasaki (2001)
\end{abstract}

\section{References}

Shibasaki, K. 2001 ApJ 557, 326-331. 


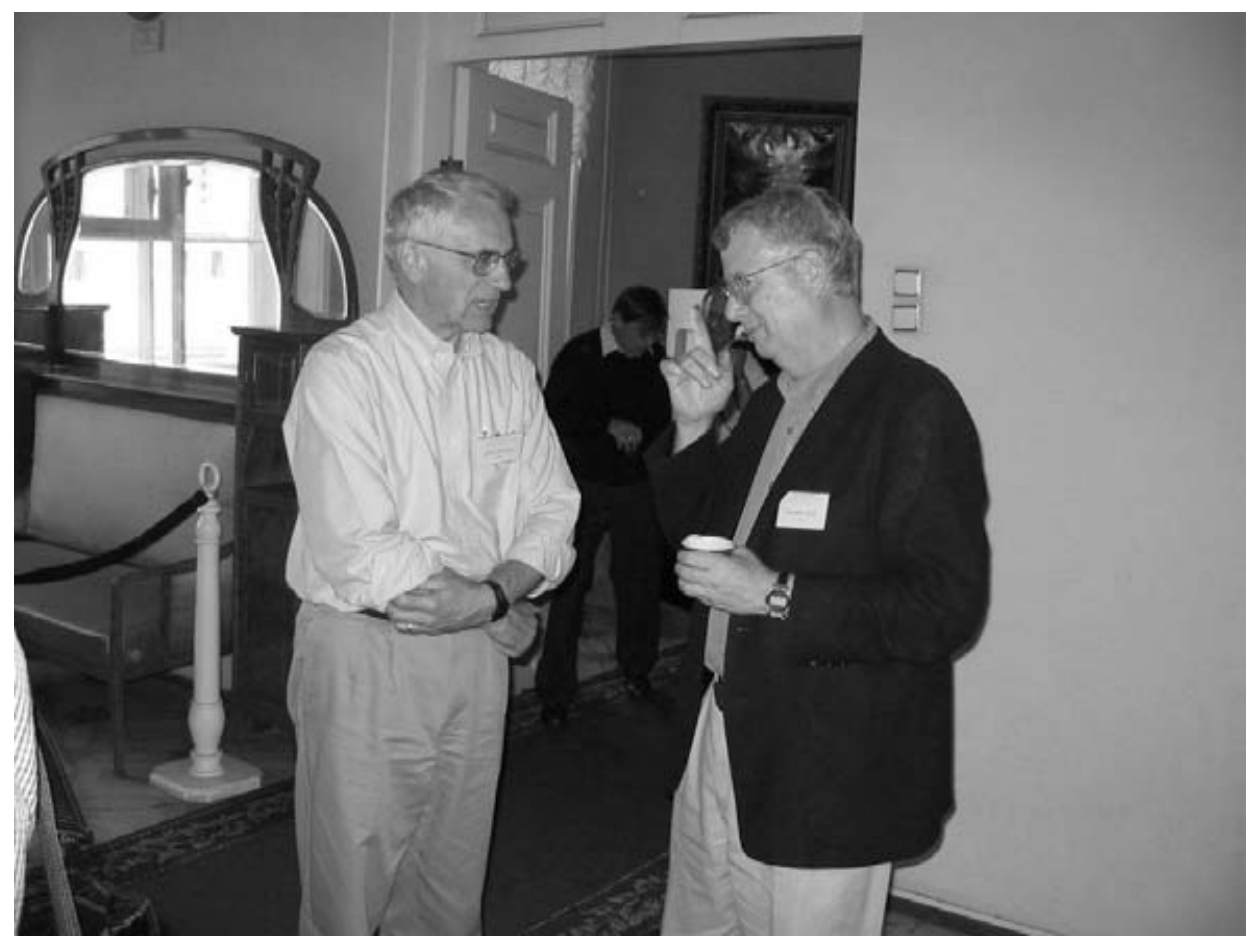

Jack Thomas (USA) and Steven Keil (USA)

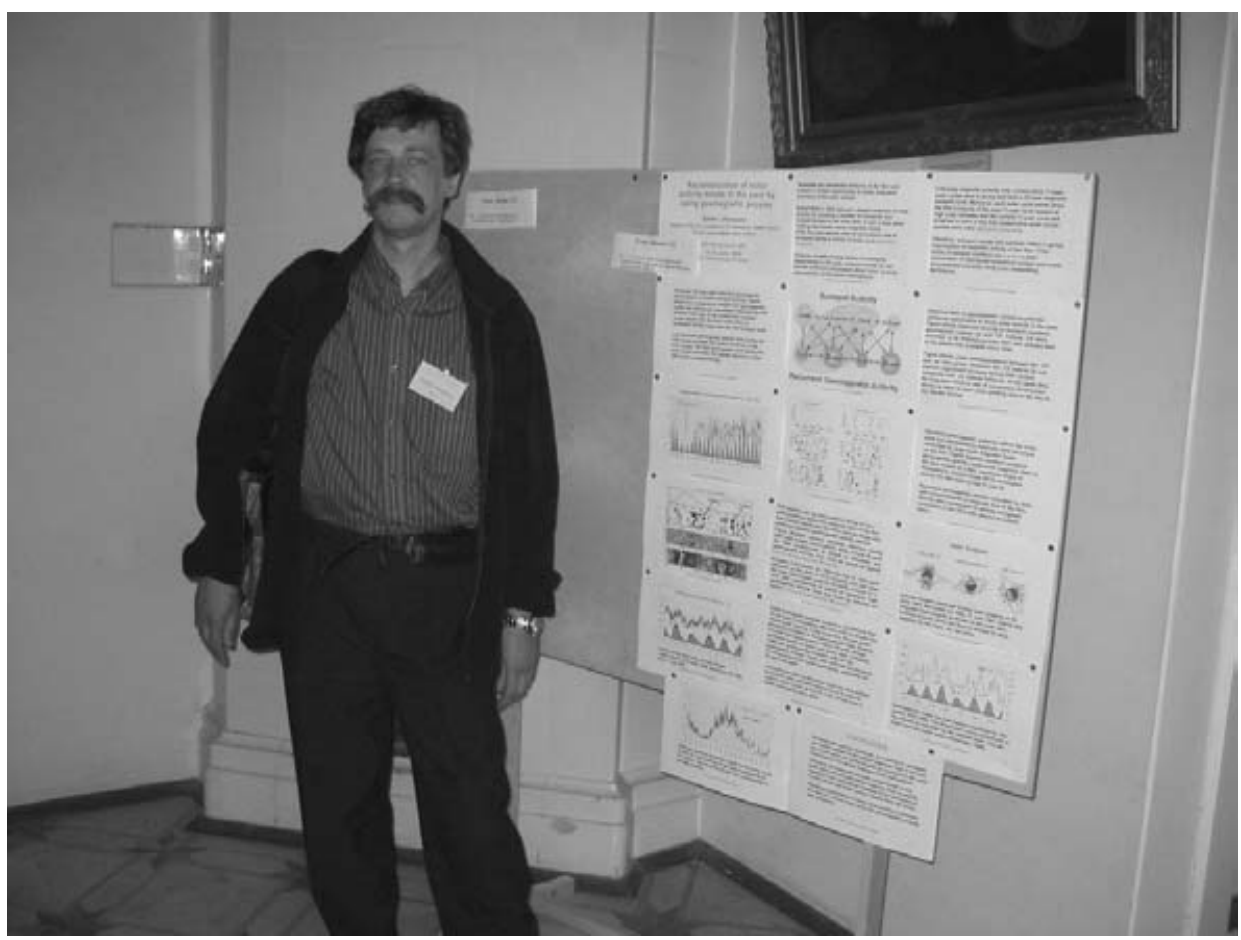

Dmitri Ponyavin (Russia) 